\title{
Otimização de Processos de Extração de Princípios Ativos de Plantas
}

\author{
Gerlon A. R. Oliveira
}

Algumas atividades biológicas associadas a plantas devem-se a grupos específicos de constituintes químicos, os princípios ativos. A extração desses compostos pode ser feita por diferentes métodos, e cada método possui variáveis de processo que podem influenciar na extração. Por isso, é necessário estabelecer as condições experimentais que possibilitem uma extração seletiva e com boa recuperação dos compostos de interesse. O delineamento experimental é uma abordagem eficiente para o planejamento dos experimentos de otimização dos processos extrativos. Neste trabalho, as principais etapas para se realizar a otimização de processos extrativos de princípios ativos de plantas são apresentadas.

Palavras-chave: extração; plantas; otimização.

Some biological activities associated with plants are due to specific groups of chemical constituents, the bioactive compounds. The extraction of these compounds can be performed by several methods, and every method has process variables that may influence the extraction. It is therefore necessary to establish the experimental conditions that allow a selective extraction and with good recovery of the compounds of interest. The experimental design is an efficient approach for planning optimization experiments of extractive processes. In this paper, the main steps to perform the optimization of extractive processes of plant bioactive compounds are presented.

Keywords: extraction; extraction; plants; optimization. 


\section{Introdução}

O homem sempre se valeu das plantas para suprimento de suas necessidades, como a alimentação e a busca da saúde física e mental. O relato de efeitos indesejados de compostos sintéticos, e a restrição ao seu uso têm aumentado a demanda por derivados de plantas com propriedades curadoras ou de manutenção da saúde ${ }^{1,2}$. A utilização de compostos provenientes de plantas, consumidas há séculos pelo homem, é vista como mais segura pela população, o que justifica o grande interesse atual da comunidade científica por estudos sobre produtos derivados de plantas.

O uso de plantas in natura ou em pó tem algumas restrições, principalmente, ligadas à variabilidade natural delas mesmas e à baixa concentração dos compostos responsáveis pela atividade desejada. A alternativa para contornar essas dificuldades consiste em submeter a planta a processos extrativos, os quais visam separar os constituintes de interesse do restante da matriz. O produto obtido, chamado de extrato, geralmente tem maior concentração de princípios ativos, maior aplicabilidade e, portanto, maior valor agregado que a matéria-prima. O problema da baixa reprodutibilidade também é contornado, pois os extratos obtidos de diferentes lotes de matérias-primas podem ser misturados, ou, ainda, diferentes quantidades de adjuvantes podem ser adicionadas a cada lote, visandose obter um extrato com concentrações padronizadas dos compostos de interesse.

Ao longo do tempo, vários métodos de extração têm sido propostos ${ }^{3,4}$. De uma forma geral, a matéria-prima pré-processada é colocada em contato com um solvente durante um período de tempo em que o solvente extrai os constituintes de interesse e, em seguida, separa-se o extrato da parte da matriz vegetal que permaneceu insolúvel. O extrato, então, pode ser utilizado tal como obtido, como é o caso de chás e tinturas, ou pode ser concentrado e/ ou $\operatorname{seco}^{5}$. Os métodos extrativos diferem uns dos outros sob vários aspectos, como o tipo de solvente utilizado (p. ex. líquidos ou fluidos supercríticos), as condições de temperatura e pressão, o tipo de agitação (p. ex. mecânica ou por ondas de ultrassom ou micro-ondas), entre outros.

A escolha do método a ser utilizado baseia-se na disponibilidade do sistema extrativo, nos custos de instalação e operação, e em questões ambientais, como a geração de resíduos, as quais se revelam mais importantes a cada dia. A escolha também depende das características que se deseja para o produto final, como a pureza do extrato ou sua compatibilidade com a matriz onde ele será adicionado.

Uma vez escolhidos os métodos de extração, há ainda que se estabelecer as condições operacionais deles. Para cada método, uma série de variáveis influencia nos custos do processo e nas características do produto final. A escolha de condições de extração inadequadas pode ocasionar baixa recuperação dos compostos de interesse e alta recuperação de constituintes indesejáveis da matriz, além de prejudicar o processo nos âmbitos econômicos e ambientais. Por isso, os processos tecnológicos de obtenção de extratos devem ser planejados e otimizados para que se estabeleçam as condições experimentais que melhor atendam aos interesses da indústria.

\section{Otimização de Processos}

O design de experimentos, ou delineamento experimental, é um procedimento estatístico eficiente para o planejamento de experimentos. Os dados obtidos permitem que se obtenham conclusões objetivas e válidas sobre a relação entre as variáveis dependentes e as independentes. O design experimental define quais serão os experimentos realizados. Uma ou mais variáveis do processo (ou fatores) são deliberadamente alteradas em cada experimento, e uma ou mais respostas são medidas. Após a realização dos experimentos, faz-se o tratamento matemático para se conhecer o efeito que a alteração das variáveis independentes produz sobre as respostas. Designs experimentais bem escolhidos permitem que se extraia grande quantidade de informação sobre o processo, a partir de reduzido número de experimentos ${ }^{6}$.

A prática de se variar um fator de cada vez pode produzir resultados enganosos, caso haja interação entre o efeito dos fatores. Por exemplo, em uma extração, usandose essa abordagem, o experimentador poderia concluir que um tempo de extração elevado é necessário para se extrair os constituintes de interesse. Em seguida, por outros experimentos, ele concluiria que uma temperatura elevada é necessária. Juntando as duas informações, ele concluiria que o processo mais adequado seria realizado 
em alta temperatura e por um tempo elevado. Entretanto, pode haver interação entre esses dois fatores, de modo que se o tempo for elevado, a temperatura pode ser baixa, ou, se a temperatura for elevada, o tempo pode ser baixo. Os designs experimentais propõem experimentos com alterações simultâneas dos fatores.

A obtenção de resultados satisfatórios, a partir de designs experimentais, envolve os seguintes passos ${ }^{6}$ :

1. Estabelecimento das respostas a serem otimizadas;

2. Escolha das variáveis independentes a serem avaliadas;

3. Escolha de um design experimental;

4. Execução do design experimental;

5. Avaliação dos dados;

6. Análise e interpretação dos resultados;

7. Uso do modelo.

Essas etapas podem ser implementadas para vários tipos de processos, inclusive para a extração de princípios ativos de plantas.

\section{Respostas Otimizadas nos Processos Extrativos}

Oprocessodeotimizaçãovisamelhoraras características do produto ou do processo e, portanto, métodos para quantificar essas características imprescindíveis. É preciso muita clareza na definição de quais sejam os interesses que se têm em vista ao se propor a otimização de um processo extrativo. Os sistemas extrativos são avaliados por meio de respostas quantitativas, que dão indícios da qualidade do extrato, e da eficiência da extração.

O rendimento total da extração é um parâmetro frequentemente utilizado na avaliação da eficiência dos processos extrativos ${ }^{7,8}$. Ele representa a relação entre a massa de extrato obtida e a massa de matéria-prima utilizada, e é calculado mediante um procedimento de perda por dessecação. Alto rendimento significa que maior quantidade de produto é obtida e, portanto, há expectativa de ganho comercial. É preciso considerar, contudo, que essa resposta não pode ser utilizada sozinha, uma vez que um alto rendimento não é indício de alta qualidade se os constituintes extraídos não são os responsáveis pela atividade atribuída à planta.

A concentração dos princípios ativos, utilizados como marcadores químicos, é seguramente o índice mais representativo da qualidade dos extratos, e do quanto o processo extrativo foi adequado. Por isso, os extratos de uso mais difundido no mundo todo, inclusive os relatados nas farmacopeias de maior alcance, são rotulados pelos teores de princípios ativos.

$\mathrm{Na}$ otimização dos processos extrativos, a concentração deve ser medida em base seca, caso contrário, os extratos obtidos utilizando-se menor razão líquido/sólido, levarão vantagem, pois uma maior quantidade de matéria-prima por unidade de volume foi submetida à extração. Portanto, um procedimento de perda por dessecação deve ser conduzido paralelamente, para que se possa estimar o rendimento da extração. De posse desse resultado, e da concentração dos marcadores no extrato líquido, é possível calcular a concentração dos marcadores no extrato, em base seca.

A concentração dos marcadores no extrato, em base seca, indica a qualidade do extrato. Em base úmida, indica a quantidade extraída da planta, ou seja, a recuperação do processo extrativo. Essa concentração também é função da qualidade da matéria-prima. Por mais que o processo extrativo seja adequado, ele é incapaz de extrair grandes quantidades de princípios ativos de uma matéria-prima pobre nesses constituintes. Um indicativo melhor da recuperação do processo extrativo é a concentração dos marcadores em relação à massa da matéria-prima utilizada.

Algumas vezes, não se tem clareza sobre quais são os constituintes químicos responsáveis pela atividade de alguma planta. Nesses casos, a resposta mais adequada é obtida testando-se o extrato obtido quanto à atividade pretendida. Essa abordagem também é útil quando a atividade se dá por mecanismos sinérgicos, em que vários constituintes contribuem para a ação pretendida, e seja difícil a quantificação de todos eles. Extratos a serem utilizados como antioxidantes geralmente são avaliados quanto a respostas ligadas à atividade antioxidante, como o conteúdo de fenóis e flavonoides, a atividade sequestradora de radicais livres, a capacidade de inibir a peroxidação lipídica, etc. ${ }^{9,10}$ Enfim, devem-se buscar respostas que deem bons indicativos sobre a qualidade do processo extrativo e do produto obtido. 


\section{Variáveis Independentes que Influenciam nos Processos Extrativos}

Por melhor que seja o processo extrativo, seu sucesso está sempre condicionado à qualidade da matéria-prima. Os métodos de obtenção e pré-processamento da matériaprima vegetal devem ser criteriosamente avaliados. A concentração dos marcadores pode variar em função de fatores genéticos, ambientais e sazonais ${ }^{11}$, o que justifica cuidado na escolha dos fornecedores da matéria-prima. $\mathrm{O}$ pré-processamento da planta inclui operações como prélavagem, secagem, e moagem ${ }^{12}$.

A secagem geralmente se dá em temperaturas elevadas, o que pode propiciar a degradação de substâncias termolábeis ${ }^{13}$ e a perda de substâncias voláteis. O controle da moagem é uma etapa crucial, pois o tamanho das partículas do pó tem forte influência no processo extrativo. A menos que ocorra aglomeração do pó durante o processo extrativo, a extração é favorecida com pós mais finos, pois a superfície de contato exposta à ação do solvente é maior ${ }^{14}$. Entretanto, a moagem acarreta custos ao processo e, portanto, é importante que se conheça o tamanho de partícula que não prejudique o processo extrativo, mas que também não onere o processo sem necessidade. A moagem também favorece a perda de constituintes termolábeis e voláteis. Após o pré-processamento e antes de ser submetida à extração, a matéria-prima fica estocada. As condições de estocagem também influenciam na concentração dos marcadores ${ }^{13}$, e devem ser estabelecidas apropriadamente.

A importância do tipo de solvente utilizado como líquido extrator é evidente, uma vez que a solubilidade, tanto dos constituintes desejáveis, quanto dos indesejáveis, é função da constante dielétrica do solvente. O desafio é encontrar um solvente, ou uma mistura de solventes, capaz de extrair, do modo mais seletivo possível, os constituintes de interesse. Outros fatores devem ser levados em conta, como a toxicidade aos técnicos das indústrias e aos consumidores, a segurança do descarte, sua disponibilidade nas quantidades necessárias, e o custo $^{15}$. Nas extrações sólido-líquido, a preferência pelas misturas etanol-água deve-se às vantagens ambientais, econômicas, e de segurança. Outra vantagem é que, dependendo da concentração alcoólica da mistura, podem-se extrair constituintes de maior ou menor polaridade. Além do tipo de solvente utilizado, o $\mathrm{pH}$ do meio é outro fator que altera o equilíbrio da extração ${ }^{9}$. Nos sistemas que utilizam fluidos supercríticos, a proporção dos solventes modificadores tem grande influência nos processos extrativos ${ }^{4}$.

O tempo de extração é um fator de grande importância nos processos extrativos. Tempos muito baixos podem ser insuficientes para permitir ao solvente extrair os compostos de interesse, enquanto que tempos muito longos podem favorecer a degradação desses ou a extração de constituintes indesejáveis, ou simplesmente representam um desperdício. O tempo de extração pode variar de poucos minutos até várias horas, ou dias inteiros ${ }^{16}$, dependendo do método extrativo e das características da matéria-prima.

A temperatura também altera o equilíbrio da extração. Temperaturas mais altas geralmente favorecem a solubilização dos constituintes. Contudo, há que se avaliar se a temperatura também não está aumentando a solubilização dos constituintes indesejados, e se os compostos de interesse não se degradam a temperaturas elevadas. O uso de solventes orgânicos de baixo ponto de ebulição em temperaturas elevadas apresenta riscos. Além disso, é preciso avaliar o impacto financeiro do aquecimento de grandes volumes de líquidos, e da instalação de sistemas de aquecimento e isolamento térmico. Portanto, o uso de temperaturas elevadas, nos processos industriais de extração, somente é justificado por grandes vantagens no processo extrativo.

A razão entre o volume de líquido extrator e a massa de sólidos da matéria-prima tem sido relatada como um fator de grande influência nas extrações ${ }^{9,17}$. Razões elevadas, geralmente, facilitam a extração, pois aumentam a solubilidade dos constituintes da planta. Por outro lado, o extrato obtido será mais diluído, o que torna o processo de concentração e secagem mais caro. Além disso, altos volumes de solvente favorecem a extração de todos os constituintes da planta, e não somente dos de interesse, o que pode originar um extrato menos concentrado. O ideal é estabelecer o menor volume de solvente capaz de extrair os constituintes de interesse, do modo mais seletivo possível. 
Existem variáveis peculiares aos diferentes sistemas de extração, as quais influenciam no processo extrativo. As extrações por ultrassom ou por micro-ondas, por exemplo, são influenciadas pela potência da radiação emitida $^{18,19}$ e a extração por fluido supercrítico é influenciada pela taxa de fluxo do fluido e pela pressão do sistema ${ }^{20}$. É preciso estudar o impacto de cada etapa com potencial influência no processo extrativo, para se determinarem as variáveis influentes.

Uma vez que já se tenham definido as respostas que se deseja avaliar (variáveis dependentes), e as variáveis com potencial influência no processo extrativo (variáveis independentes), o objetivo da otimização é encontrar as condições em que as variáveis independentes devem ser configuradas para que as variáveis dependentes apresentem os resultados mais apropriados. Isso é feito de modo empírico, pois o efeito das variáveis independentes sobre as respostas é complexo demais para ser determinado com precisão apenas pela teoria.

\section{Designs Experimentais Utilizados para Avaliação de Processos Extrativos}

De acordo com o objetivo que se tem em vista, diferentes designs experimentais podem ser escolhidos. Quando não se sabe quais são as variáveis importantes no processo extrativo, recorre-se a um experimento de triagem. Se o experimentador suspeita que um número de variáveis menor que cinco é importante, recomenda-se a utilização de experimentos fatoriais completos ou fracionários. Para a verificação prévia de um número de variáveis igual ou superior a cinco, recomendam-se experimentos fatoriais fracionários ou o design de Plackett-Burman ${ }^{6}$. Esses designs permitem avaliar muitas variáveis mediante poucos experimentos. Anastácio e Carvalho, por exemplo, avaliaram a influência de nove variáveis mediante doze experimentos e concluíram que apenas duas delas influenciavam na extração de compostos fenólicos da casca de batata ${ }^{21}$.

A avaliação de um número alto de variáveis por meio de poucos experimentos, obviamente, tem suas limitações. Experimentos dessa natureza dão informações apenas suficientes para se determinar se uma variável é ou não importante. Não é possível chegar-se a um valor ótimo para as respostas, utilizando esses experimentos, exceto por um acaso.

O número de variáveis importantes nos processos extrativos não é tão alto, e é possível determiná-las previamente, pelo conhecimento do processo. Em caso de dúvidas, um planejamento de triagem pode ser realizado, ao final do qual geralmente se constata que o número de variáveis importantes é reduzido. Essas variáveis, então, são estudadas em um novo planejamento experimental. Além disso, por questões de ordem prática, algumas variáveis, ainda que reconhecidamente importantes, não são avaliadas . Por exemplo, para evitar o aumento de custos de produção, ou por questões de segurança, pode-se escolher extrair à temperatura ambiente, e não avaliar o efeito da temperatura, embora se saiba que ele é, provavelmente, relevante. Por isso, grande parte dos estudos sobre otimização de extrações avalia o efeito de, no máximo, quatro variáveis independentes ${ }^{7,10,18,22}$.

A otimização de um processo com um número igual ou inferior a quatro variáveis independentes é feita, comumente, pela metodologia de superfície de resposta. Dentro dessa abordagem, diversos designs experimentais são possíveis. Eles permitem que se obtenham modelos quadráticos ou, menos usualmente, cúbicos $^{6}$. Muitas das respostas, comumente avaliadas em processos extrativos, não dependem linearmente das variáveis independentes. A recuperação dos marcadores, por exemplo, é baixa quando a razão líquido-sólido é muito baixa, porque não ocorre completa solubilização. Aumentando-se a razão líquido-sólido, a recuperação atinge um valor ótimo, quando a maior parte dos marcadores é extraída. Em seguida, ocorre uma estabilização, e o aumento do volume de líquido extrator não ocasiona incremento na extração, pois os marcadores já foram completamente extraídos. Da mesma forma, concentrações alcoólicas abaixo ou acima de certo valor podem não extrair adequadamente um composto. Portanto, em processos extrativos, designs capazes de prever um comportamento de estabilização, ou de aumento seguido de diminuição das respostas, são necessários. Os designs experimentais usados na metodologia de superfície de resposta têm se mostrado adequados a 


\section{Artigo Convidado 2}

tais finalidades. A Figura 1 ilustra uma superfície de resposta genérica, a qual foi montada utilizando termos lineares e quadráticos. Como se pode observar, a estabilização da resposta com o aumento da variável codificada 1 pôde ser prevista pelo modelo.

Os designs mais utilizados na metodologia de superfície de resposta são os de delineamento composto central ${ }^{9,10,20,23,24}$ e os de Box Behnkenn ${ }^{25-27}$. Estes últimos requerem um número de experimentos menor, quando três ou quatro fatores são avaliados, porém, apresentam regiões experimentais de baixo poder de predição. Os designs usando delineamento composto central têm sido mais utilizados na literatura de extrações de princípios ativos de plantas.

Os designs experimentais propostos na literatura são roteiros que indicam os níveis em que cada fator deve estar, na série de experimentos a serem realizados. Esses níveis são codificados para normalizar as variáveis de magnitudes diferentes. Após a codificação, todas as variáveis estarão contidas em um domínio experimental com a mesma variação. Os designs propõem os níveis codificados, mas cabe ao experimentador escolher a faixa de valores a se trabalhar. Em um trabalho da literatura, variações de pressão de 450 para $550 \mathrm{MPa}$, de concentração de etanol na mistura hidroalcoólica de 50 para $60 \%$, e de razão líquido-sólido de 20 para $25 \mathrm{~mL} / \mathrm{g}$, foram normalizadas, de modo que todos representassem uma unidade de variação, nas variáveis codificadas ${ }^{17}$. O experimentador deve conhecer o sistema extrativo com o qual trabalha, para que não faça codificações incoerentes.

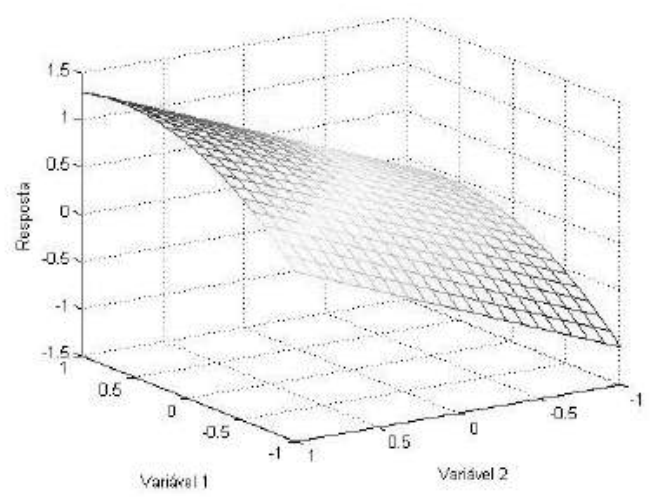

Figura 1. Ilustração de uma superfície de resposta para otimização de um processo extrativo. As variáveis independentes estão um domínio experimental codificado. Equação usada para montar a superfície: Resposta $=0,5+0,5 *($ Variável 1$)+0,7 *($ Variável 2$)-0,4 *(\text { Variável } 1)^{2}$.
A escolha do domínio experimental é muito importante para se obter modelos confiáveis. Antes de se otimizar, é necessário saber qual a faixa de valores que as variáveis independentes podem assumir, de modo que o processo não saia dos limites da viabilidade técnica e econômica. A escolha da faixa em que cada variável será avaliada é feita a partir de um estudo prévio, o qual conta com levantamento bibliográfico e com experimentos preliminares. O ponto ótimo deve estar dentro do domínio experimental testado, para que ele seja predito através de uma interpolação do modelo, e não de uma extrapolação. Fora do domínio experimental testado, pode haver efeitos que não foram previstos.

O ponto ótimo, preferencialmente, não deve estar nos níveis extremos do domínio experimental testado. Se o modelo indica que a resposta ainda está melhorando no limite da região experimental testada, o ideal é realizar um planejamento na nova região. Entretanto, limitações técnicas podem impedir essa mudança. Um modelo pode indicar, por exemplo, que uma concentração alcoólica superior à testada no planejamento experimental aumentaria a extração de um princípio ativo. Se a mistura com máxima concentração testada foi o etanol absoluto, então, não será possível modificar o domínio experimental dessa variável.

\section{EXECUÇÃO DO DESIGN EXPERIMENTAL NOS PROCESSOS EXTRATIVOS}

Os designs experimentais indicam quais os experimentos a serem realizados, mas a ordem de realização deve ser aleatória. Isso porque pode haver algum fator, ignorado pelo experimentador, variando em função do tempo ou da ordem de realização do experimento. Nesse caso, se os experimentos são realizados aleatoriamente, o efeito do fator ignorado é distribuído aleatoriamente entre os experimentos. Outra recomendação é a de que os experimentos sejam realizados de forma independente. Após cada experimento, as condições iniciais dos equipamentos devem ser restabelecidas, também objetivando à aleatoriedade dos experimentos. Recomenda-se, ainda, que se disponha de tempo e material a mais do que o previsto, para o caso de eventos inesperados ${ }^{6}$. No caso de ser necessário repetir algum experimento, 
caso não se disponha de matéria-prima suficiente, pode-se perder um grande trabalho.

\section{Avaliação dos Dados}

A primeira observação a ser feita, após a realização dos experimentos, é quanto à estabilidade do processo extrativo durante a execução do design experimental. Os designs contam com replicatas de experimentos, para que se estime o erro experimental. Não é bom que as replicatas tenham um desvio padrão relativo elevado, pois, nesse caso, os efeitos podem se confundir com os erros. A aleatoriedade da variação das respostas das replicatas, durante o tempo, também indica a estabilidade do processo ${ }^{6}$. Após avaliar a estabilidade do processo, passa-se à avaliação dos modelos de previsão.

Uma forma de se avaliar a adequação dos modelos, é pela análise das diferenças entre os resultados experimentais e os preditos, ou seja, dos resíduos. Os resíduos não são explicados pelo modelo, e, por serem uma forma de erro, devem seguir a distribuição normal, com média zero. Devem, ainda, distribuirem-se de forma aleatória em relação à ordem em que os experimentos foram realizados, à magnitude da resposta predita, e à configuração das variáveis independentes envolvidas na predição ${ }^{6}$. Gráficos da distribuição dos resíduos são ferramentas importantes na avaliação da adequação dos modelos de previsão.

Os modelos também são avaliados quanto à capacidade de fazerem previsões acertadas. A análise de variância indica que um bom modelo tem uma regressão significativa e uma falta de ajuste não significativa. Os resíduos devem ser compostos, em grande parte, pelo erro experimental, e não pela falta de ajuste. A significância das variáveis do modelo é medida pelo valor de p dessas, que indica a probabilidade de sua influência ser casual. Após determinarem-se as variáveis não significativas do modelo, essas devem ser descartadas, e um novo modelo calculado.

Quanto mais próximo de 1 o valor do coeficiente de variação $\mathrm{R}^{2}$ do modelo, melhor o seu ajuste às respostas obtidas experimentalmente ${ }^{28}$. Entretanto, o $\mathrm{R}^{2}$ pode estar alto por estar inserido um grande número variáveis no modelo, dentre as quais, algumas podem não ser significativas. A inserção de variáveis a um modelo, ainda que não significativas, aumenta o valor do $\mathrm{R}^{2}$. $\mathrm{O}$
$\mathrm{R}^{2}$ ajustado é um parâmetro de avaliação mais adequado, pois seu valor diminui, caso se adicionem ao modelo variáveis não significativas ${ }^{6}$.

O objetivo do planejamento experimental pode ser otimizar mais de uma resposta do processo extrativo, como a recuperação de mais de um princípio ativo ${ }^{23}$. Nesse caso, funções de desejabilidade são empregadas. Esse método consiste em transformar cada resposta em uma nova resposta, cujo valor varia entre zero e 1 . Quanto mais próxima de 1, mais desejável a resposta é. As respostas que se quer otimizar, após transformadas em desejabilidades individuais, são combinadas em uma desejabilidade global única, que geralmente é a média geométrica de todas ${ }^{29}$. Assim, otimiza-se a desejabilidade global, como se otimizaria uma única resposta.

\section{Análise e Interpretação dos Resultados}

Uma vez que se tenham obtidos modelos adequados, o próximo passo é avaliá-los para saber os níveis em que as variáveis independentes devem ser fixadas, visando a uma resposta mais adequada. O ponto ótimo, ou região experimental ótima, é mais facilmente visualizado por meio de gráficos, ou superfícies de resposta. Quando mais que duas variáveis influenciam significativamente em uma resposta, não é possível construir uma superfície única para apresentar toda a informação, pois haveria mais que três dimensões. Nesses casos, costumam-se apresentar os gráficos de superfície com uma resposta em função de duas variáveis independentes, ignorando-se as demais. Atribui-se o valor zero ao nível das variáveis não apresentadas. Um processo extrativo com duas respostas e três variáveis independentes gera seis superfícies de resposta. Uma alternativa para diminuir o número de superfícies é a apresentação de gráficos em quatro dimensões, em que, em um cubo, cada eixo representa uma variável independente, e a resposta é representada por uma escala de cores (a Figura 2 é um exemplo desses gráficos em 4 dimensões). Entretanto, nem sempre essa estratégia é possível, pois se o ponto ótimo encontrarse no interior do cubo, ele pode não ser enxergado. De qualquer forma, é bom que se avaliem os modelos não só pela forma gráfica, como também pelo entendimento matemático da equação. 


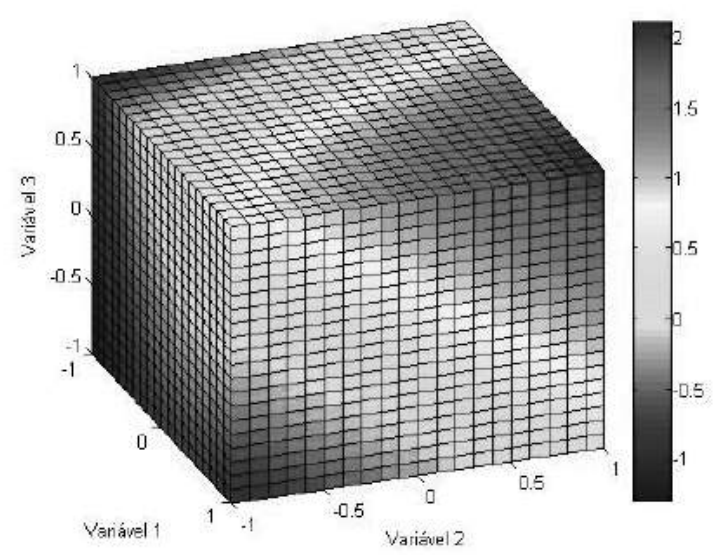

Figura 2. Ilustração de um gráfico em 4 dimensões para otimização de um processo extrativo. As variáveis independentes estão um domínio experimental codificado. A escala de cores indica a variação das respostas. Equação usada para montar o gráfico: Resposta $=0.5+0.5^{*}$ (Variável 1) + 0,7* (Variável 2) + 0,5* (Variável 3) - 0,4* (Variável $1)^{2}+0,3 *($ Variável 1$) *($ Variável 3$)$.

Os modelos dão uma indicação das regiões mais adequadas, mas a escolha das condições extrativas a serem empregadas em escala industrial engloba outros fatores, que devem ser criteriosamente avaliados. Se uma região não contiver o ponto ótimo do modelo, mas apresentar valores próximos a ele, e, por outro lado, representar benefícios de ordem prática, econômica e ambiental, ela provavelmente será escolhida. No contexto acadêmico, isso talvez não seja tão importante, mas seguramente o é nos âmbitos da indústria e do mercado.

\section{Uso do Modelo}

Se o modelo indicar que a condição ótima pode estar fora do domínio experimental avaliado, convém realizar um novo planejamento nesta região. Caso contrário, pode-se validar o modelo, pela realização de um experimento nas condições escolhidas, para se comprovar sua previsão. Se os experimentos foram realizados em escala laboratorial, procede-se ao aumento de escala, para que, por fim, o método de extração dos constituintes de interesse das plantas possa ser utilizado na indústria.

\section{Referências}

1. Wanasundara, U. N.; Shahidi, F.; Food Chem. 1998, 63, 335.

2. Vitaglione, P.; Fogliano, V.; J. Chromatogr. B. Analyt. Technol. Biomed. Life Sci. 2004, 802, 189.

3. Carvalho, R. N.; Moura, L. S.; Rosa, P. T. V.; Meireles, M. A. A.; J. Supercrit. Fluids 2005, 35, 197.

4. Herrero, M.; Plaza, M.; Cifuentes, a; Ibáñez, E.; J. Chromatogr. A 2010, 1217, 2512.

5. Azmir, J.; Zaidul, I. S. M.; Rahman, M. M.; Sharif, K. M.; Mohamed, a.; Sahena, F.; Jahurul, M. H. a.; Ghafoor, K.; Norulaini, N. a. N.; Omar, a. K. M.; J. Food Eng. 2013, 117, 426.

6. NIST SEMATECH Engineering Statistics e-Handbook; 2012

7. Rezzoug, S. A.; Boutekedjiret, C.; Allaf, K.; J. Food Eng. 2005 , 71,9 .

8. Rodríguez-Rojo, S.; Visentin, A.; Maestri, D.; Cocero, M. J.; J. Food Eng. 2012, 109, 98.

9. Dorta, E.; Lobo, M. G.; González, M.; Food Bioprocess Technol. 2011, 6, 1067.

10. Singh, B.; Sharma, H. K.; Sarkar, B. C.; J. Food Sci. Technol. 2011, 49, 294.

11. Hidalgo, P. J.; Ubera, J. L.; Tena, M. T.; Valcárcel, M. ;J. Agric. Food Chem. 1998, 46, 2624.

12. Ong, E. S.; J. Chromatogr. B. Analyt. Technol. Biomed. Life Sci. 2004, 812, 23.

13. Mulinacci, N.; Innocenti, M.; Bellumori, M.; Giaccherini, C.; Martini, V.; Michelozzi, M.; Talanta 2011, 85, 167.

14. Sari, F.; Velioglu, Y. S.; J. Food Compos. Anal. 2011, 24, 1130.

15. Albu, S.; Joyce, E.; Paniwnyk, L.; Lorimer, J. P.; Mason, T. J.; Ultrason. Sonochem. 2004, 11, 261.

16. Pérez-Serradilla, J. A.; Luque de Castro, M. D.; Food Chem. 2011, 124, 1652.

17. Xi, J.; Wang, B.; Food Bioprocess Technol. 2012, 6, 2538.

18. Chavan, Y.; Singhal, R. S.; Innov. Food Sci. Emerg. Technol. 2013, 17, 106.

19. Song, J.; Li, D.; Liu, C.; Zhang, Y.; Innov. Food Sci. Emerg. Technol. 2011, 12, 282.

20. Shao, P.; Sun, P.; Ying, Y.; Food Bioprod. Process. 2008, 86, 227.

21. Anastácio, A.; Carvalho, I. S.; Ind. Crops Prod. 2013, 43, 99.

22. Elksibi, I.; Haddar, W.; Ben Ticha, M.; Gharbi, R.; Mhenni, M. F.; Food Chem. 2014, 161, 345.

23. Yang, B.; Liu, X.; Gao, Y.; Innov. Food Sci. Emerg. Technol. 2009, 10, 610 . 
24. Ghafoor, K.; Choi, Y. H.; Jeon, J. Y.; Jo, I. H.; J. Agric. Food Chem. 2009, 57, 4988.

25. Swamy, G. J.; Sangamithra, A.; Chandrasekar, V.; Dye. Pigment. 2014, 111, 64.

26. Ahmad, A.; Alkharfy, K. M.; Wani, T. A.; Raish, M. ;Int. J. Biol. Macromol. 2014

27. Prakash Maran, J.; Manikandan, S.; Thirugnanasambandham, K.; Vigna Nivetha, C.; Dinesh, R.; Carbohydr. Polym. 2013, 92, 604.

28. Teófilo, R.; Ferreira, M.; Quim. Nova 2006, 29, 338.

29. Derringer, G.; J. Qual. Technol. 1980, 12, 214.

\section{Gerlon A. R. Oliveira}

Universidade Federal de Goiás, CEP 74001-970, Caixa Postal 131, Goiânia, GO, Brasil. Instituto de Química.

SENAI - GO, Faculdade de Tecnologia Roberto Mange. Rua Engenheiro Roberto Mange, n 239. Bairro Jundiaí - CEP 75113-630 Anápolis, GO, Brasil.

E-mail: gerlonalmeida@gmail.com 
